\title{
Caracterização de Filtros em Linhas de Microfita com Planos de Terra Fractais
}

\author{
Rossana Moreno Santa Cruz, Tarcílio Cavalcanti da Silva, Paulo Henrique da Fonseca Silva \\ e Adaildo Gomes D’Assunção
}

\begin{abstract}
Resumo - Este trabalho investiga o comportamento em freqüência de filtros projetados e construídos em linhas de microfita, utilizando estruturas periódicas fractais no plano de terra. O fractal denominado "tapete" de Sierpinski, gerado a partir de três iterações, foi utilizado para este propósito. $O$ objetivo é oferecer contribuições aos projetos em microfita de estruturas compactas e multibanda, fabricadas a partir de figuras fractais e com aplicações na faixa de freqüências ISM (Industrial Scientific \& Medical). Pretende-se também investigar a utilidade dos fractais para a miniaturização de antenas em microfita, bem como verificar suas propriedades eletromagnéticas multibanda e os benefícios decorrentes destas aplicações.
\end{abstract}

Palavras-chaves - Filtros, linhas de microfita, geometria fractal, freqüência de ressonância.

Abstract - This work investigates the frequency behavior of the design of microstrip transmission line filters, using fractal periodic structures as the ground plane. The fractal geometry in use is the so called Sierpinski Carpet which was designed based on three iterations. The goal of this work is to contribute with the microstrip designs of multiband and compact structures, made of fractal geometries and applied on ISM (Industrial, Scientific \& Medical) band. Another purpose is to investigate the use of the fractals on the miniaturization of microstrip antennas, analyzing their multiband electromagnetic characteristics and the benefits due to their applications.

Keywords - Filters, microstrip lines, fractal geometry, resonant frequency.

\section{INTRODUÇÃO}

Os dispositivos planares em linhas de microfita apresentam características desejáveis para aplicações aeroespaciais e em modernos sistemas de comunicações móveis; são exemplos de tais características: dimensões reduzidas, baixo custo de fabricação, baixo peso e fácil integração com circuitos impressos.

Rossana Moreno Santa Cruz, Adaildo Gomes D’Assunção, Universidade Federal do Rio Grande do Norte, Departamento de Engenharia Elétrica, Natal - RN. E-mails: mscrossana@gmail.com, adaildo@ct.ufrn.br. Paulo Henrique da Fonseca Silva e Tarcílio Cavalcanti da Silva, Centro Federal de Educação Tecnológica da Paraíba, Grupo de Telecomunicações e Eletromagnetismo Aplicado, João Pessoa - PB. E-mails: henrique@cefetpb.edu.br, tarcilio@gmail.com.

Este trabalho foi parcialmente financiado pelo CNPq.
A largura de banda estreita e o casamento de impedância são algumas das principais desvantagens destes tipos de dispositivos. Durante as últimas décadas, vários trabalhos considerando o aumento da largura de banda de antenas em microfita têm sido publicados.

Uma técnica particular caracterizada pela simplicidade de implementação é a utilização de um alimentador por acoplamento eletromagnético cuja ponta de prova coaxial é em forma de L (L-Probe) ou H (H-Probe) em [1].

A miniaturização de equipamentos eletrônicos é uma das principais tendências em sistemas de comunicação modernos.

Uma das técnicas disponíveis na literatura, utilizada para se reduzir as dimensões de antenas e filtros, consiste na aplicação de geometrias fractais. Os fractais são formas geométricas com padrões complexos que se repetem infinitamente, mesmo sendo estas formas limitadas a uma região finita.

Um filtro fractal pode ser criado utilizando-se figuras fractais, pois as qualidades inerentes destas geometrias permitem a produção desse dispositivo, com elevado desempenho e com uma redução no tamanho de cerca de 50 a 75 \% em relação aos tipos tradicionais [2].

Este trabalho investiga o comportamento em freqüência de filtros construídos em linhas de microfita, com plano terra substituído por estruturas periódicas cujas células são baseadas nos fractais de Sierpinski. Especificamente, utilizou-se o fractal denominado "tapete" de Sierpinski [3], para o qual consideraram-se três níveis diferentes. As estruturas foram construídas e submetidas à medição, para análise e coleta de dados. Os resultados foram obtidos através de medições de parâmetros de espalhamento, como os coeficientes de transmissão $\left(\mathrm{S}_{21}\right)$ e reflexão $\left(\mathrm{S}_{11}\right)$. Neste sentido, observou-se o comportamento em freqüência dos filtros abordados e a influência da introdução da geometria fractal.

\section{GEOMETRIA FRACTAL}

Atualmente, os fractais são aplicados em muitos ramos da ciência e tecnologia, desde a biologia até a engenharia. Por exemplo, a união entre o eletromagnetismo e a geometria fractal originou a área de pesquisa denominada eletrodinâmica fractal. Nesta área, as propriedades únicas da geometria fractal mostram-se úteis para a construção de 
filtros, superfícies seletivas em freqüência e antenas, possibilitando soluções práticas para uma variedade de aplicações na banda ISM.

A geometria Euclidiana trata de objetos geométricos com formas regulares, como o ponto, as curvas e as superfícies, que são caracterizados em termos de suas dimensões topológicas: 1, 2 e 3, respectivamente. Contudo, há muitas formas irregulares encontradas na natureza que estão fora da abrangência da geometria Euclidiana, tais como: superfícies de nuvens e montanhas, linhas costeiras, raízes e ramos de árvores, nervos e vasos do corpo humano [4]. Por definição, um fractal é um conjunto para o qual a dimensão de Hausdorff-Besicovitch estritamente excede a dimensão topológica. Portanto, as formas fractais possuem dimensionalidade superior às formas Euclidianas, ocupando de forma mais eficiente uma área ou volume finito [5]. O termo fractal foi introduzido em 1975 pelo matemático Benoit Mandelbrot, referindo-se a objetos construídos recursivamente, onde um aspecto do objeto limite é infinito e outro é finito [6]. A dimensão fractal, a auto-semelhança, o preenchimento de espaço e a construção iterativa são propriedades comuns aos fractais [5].

A dimensão fractal de um conjunto pode ser calculada através de vários métodos. Para o caso da geometria quadrada considerada, utilizou-se a dimensão de Homotetia ou AutoSimilaridade. Esta propriedade consiste em obter réplicas menores da figura, decorrentes de sua divisão ou ampliação. Por exemplo, um segmento de reta pode ser dividido em $p$ partes iguais, semelhantes ao segmento original, porém reduzidas, em uma certa razão $r$. O número $n$ de segmentos obtidos tem relação com a razão de semelhança $r$ da seguinte forma:

$$
n=\frac{1}{r}
$$

Como ilustração, a Fig. 1 apresenta uma reta dividida em quatro partes iguais. A razão de semelhança é, portanto, 1/4. Em seguida, é feita uma divisão de cada um de seus lados em $p$ partes iguais. Obtém-se $p^{2}$ segmentos de reta semelhantes ao original. Logo, $p^{2}=n$. Relacionando $r$ e $n$, obtém-se:

$$
n=\frac{1}{r^{2}}
$$

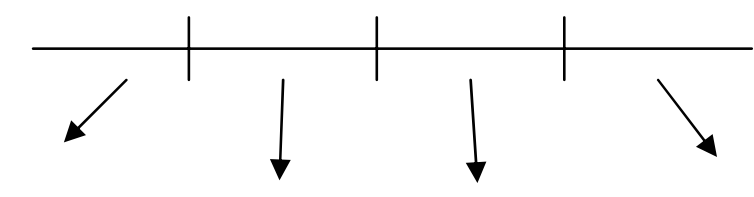

Fig. 1. Segmento de reta dividido.

A geometria fractal utilizada no trabalho é ilustrada na Fig. 2. Verifica-se que a figura geradora inicial é um quadrado cujos lados foram divididos em 3 partes iguais, originando 9 outros quadrados ( $1^{\text {a }}$ iteração). A divisão prossegue da mesma forma até a $3^{\text {a }}$ iteração, como o ilustrado na Fig. 3, sempre obedecendo a uma razão de semelhança de 1/3.

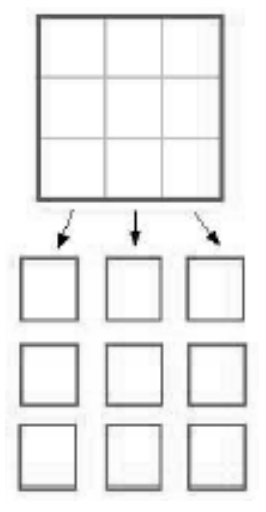

Fig. 2. Quadrado dividido (1 $1^{\mathrm{a}}$ iteração).

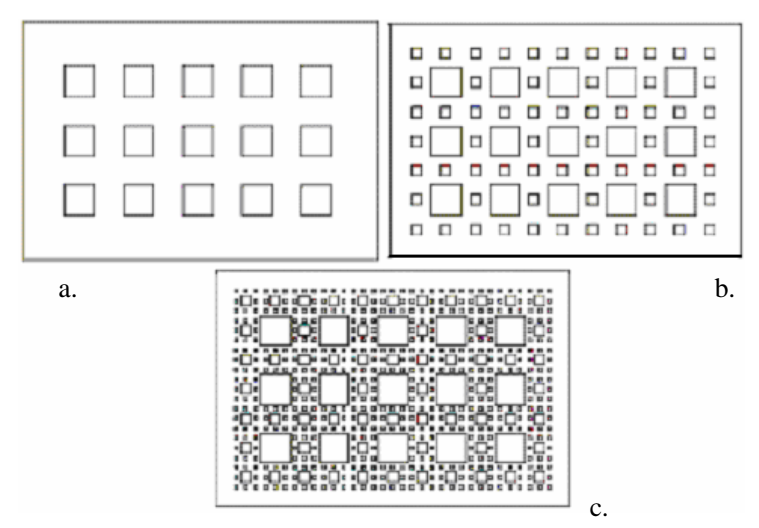

Fig. 3. Geometria fractal de Sierpinski : (a) $1^{\mathrm{a}}$, (b) $2^{\mathrm{a}}$ e (c) $3^{\mathrm{a}}$ iterações.

\section{ANÁLISE E PROCEDIMENTO DE MEDIÇÃO}

Para a obtenção do valor da largura da fita $w$ com uma impedância de entrada $Z_{0}=50 \Omega$, foram utilizados os modelos tradicionais de análise e síntese de linhas de microfita [7], de acordo com as equações (3)-(10). A partir dos cálculos realizados, foi obtido o valor de $w=2,87 \mathrm{~mm}$. A impedância característica da linha de transmissão é obtida através das equações (1) e (3), de acordo com a relação $w / h$, encontrada.

$$
Z_{0}=\frac{60}{\left(\varepsilon_{\text {eff }}\right)^{1 / 2}} \ln \left(\frac{8 h}{w}+0,25 \frac{w}{h}\right) \text {, se } w / h<1
$$

em que a permissividade efetiva ( $\left.\varepsilon_{\text {eff }}\right)$ é dada por:

$$
\varepsilon_{e f f}=\frac{\varepsilon_{r}+1}{2}+\frac{\varepsilon_{r}-1}{2}\left[\left(1+\frac{12 h}{w}\right)^{-1 / 2}+0,041\left(1-\frac{w}{h}\right)^{2}\right]
$$




$$
\begin{gathered}
Z_{0}=\frac{120 \pi}{\left(\varepsilon_{\text {eff }}\right)^{1 / 2}} \frac{1}{\left[\frac{w}{h}+1,393+0,667 \ln \left(\frac{w}{h}+1,4444\right)\right]}, \\
\text { e } \quad \varepsilon_{\text {eff }}=\frac{\delta_{\tau}+1}{2}+\frac{\varepsilon_{\tau}-1}{2}\left(1+12 \frac{h}{w}\right)^{-1 / 2}
\end{gathered}
$$

Definindo o projeto, tem-se:

$$
\frac{w}{h}=\frac{8 \exp (A)}{\exp (2 A)-2}, \text { se } \mathrm{A}<1,52
$$

ou, para $\mathrm{A} \geq 1,52$ :

$$
\frac{w}{h}=\frac{2}{\pi}\left\{B-1-\ln (2 B-1)+\frac{\varepsilon_{\tau}-1}{2 \varepsilon_{\tau}}\left[\ln (B-1)+0,39-\frac{0,61}{\varepsilon_{\tau}}\right]\right\}
$$

em que

$$
\begin{gathered}
\mathrm{A}=\frac{\mathrm{Z}_{0}}{60}\left(\frac{\varepsilon_{\tau}+1}{2}\right)^{1 / 2}+\frac{\varepsilon_{\tau}-1}{\varepsilon_{\tau}+1}\left(0,23+\frac{0,11}{\varepsilon_{\tau}}\right) \\
\mathrm{B}=\frac{377 \pi}{2 \mathrm{Z}_{0}\left(\varepsilon_{\tau}\right)^{1 / 2}}
\end{gathered}
$$

O procedimento de construção e medição dos filtros projetados foi baseado no descrito em [8]. Para o experimento, foi utilizada inicialmente uma placa de cobre dupla face (24 cm de largura por $12 \mathrm{~cm}$ de altura), especificada na Fig. 4. O substrato dielétrico utilizado foi a fibra de vidro, de permissividade elétrica relativa $\varepsilon_{\mathrm{r}}=4,4 \mathrm{e}$ espessura $h=1,578 \mathrm{~mm}$. O plano terra foi substituído por uma estrutura periódica cujas células são os fractais de Sierpinski, cujas dimensões são mostradas na Fig. 5. A Fig. 6 mostra uma fotografia do primeiro protótipo construído.

Além deste protótipo, também foi construído um outro, para a análise da variação do tamanho da fita condutora de excitação, com relação à perda de retorno e à largura de banda obtida. Neste caso, utilizou-se o mesmo substrato dielétrico de fibra de vidro, mas a placa possui dimensões de $28 \mathrm{~cm}$ de largura por $28 \mathrm{~cm}$ de altura. Cada elemento possui as mesmas dimensões apresentadas na Fig. 5, exceto pela sua periodicidade, que é de $35 \mathrm{~mm}$. A estrutura funciona como uma antena cujo plano terra é o "tapete" de Sierpinski. Vale salientar que, neste caso, os elementos não formam um plano de terra contínuo, sendo a energia transmitida através de acoplamento eletromagnético entre as células da estrutura. Uma foto deste novo protótipo construído é apresentada na Fig. 7. Os resultados obtidos através das medições são mostrados e descritos na seção IV.

Os testes e as medições foram realizados nos laboratórios do CEFET-PB. O equipamento utilizado para a coleta dos dados foi o analisador de redes vetorial - modelo N5230A PNA-L, da Agilent Technologies - que opera na faixa de 300 $\mathrm{KHz}$ a 13,5 GHz. Além dele, foram utilizados dois cabos Agilent 85132D, dois conectores tipo PCB, modelo 82_SMA50-0-1/111_N, duas transições PC7-N e duas outras N-N. Uma foto do set up de medição é mostrado na Fig. 8.

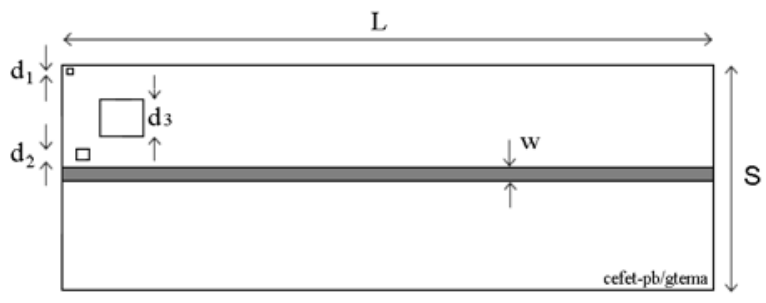

Fig. 4. Dimensões do filtro projetado em linha de microfita sobre plano terra fractal: $\mathrm{L}=120 \mathrm{~mm}, \mathrm{~S}=80 \mathrm{~mm}, \mathrm{w}=2,82 \mathrm{~mm}, \mathrm{~d}_{1}=1,11 \mathrm{~mm}, \mathrm{~d}_{2}=3,33$ $\mathrm{mm}, \mathrm{d}_{3}=10 \mathrm{~mm}$.

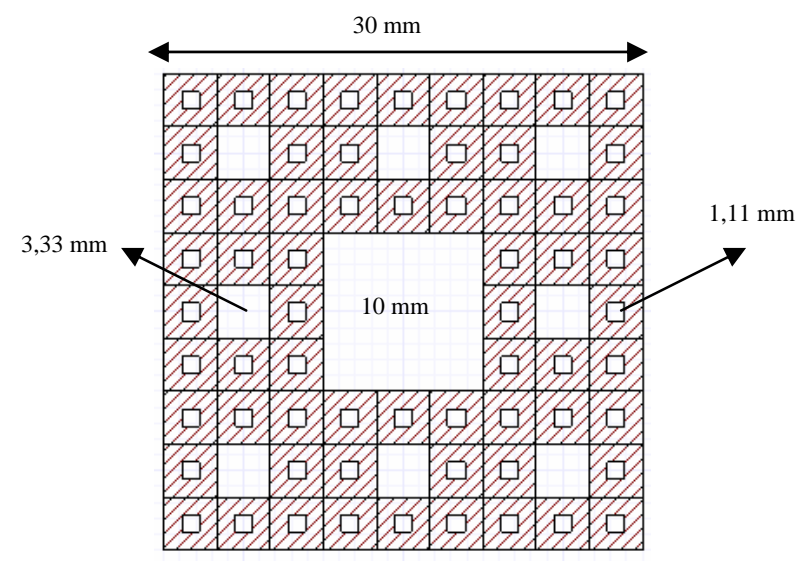

Fig. 5. Dimensões de um elemento da estrutura periódica fractal.

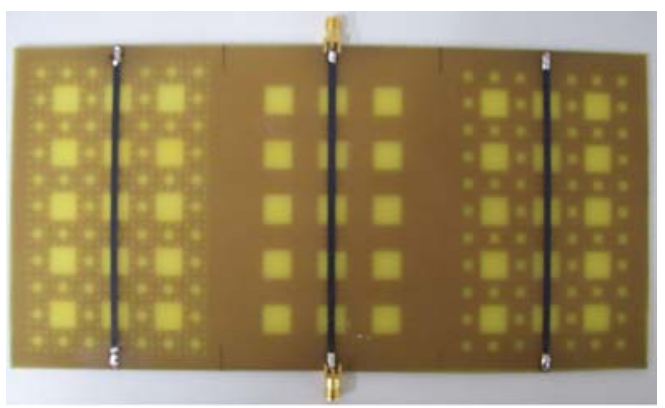

Fig. 6. Primeiro protótipo construído. 


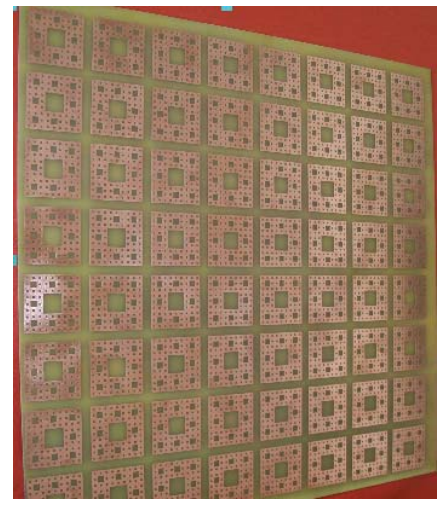

Fig. 7. Segundo protótipo construído.

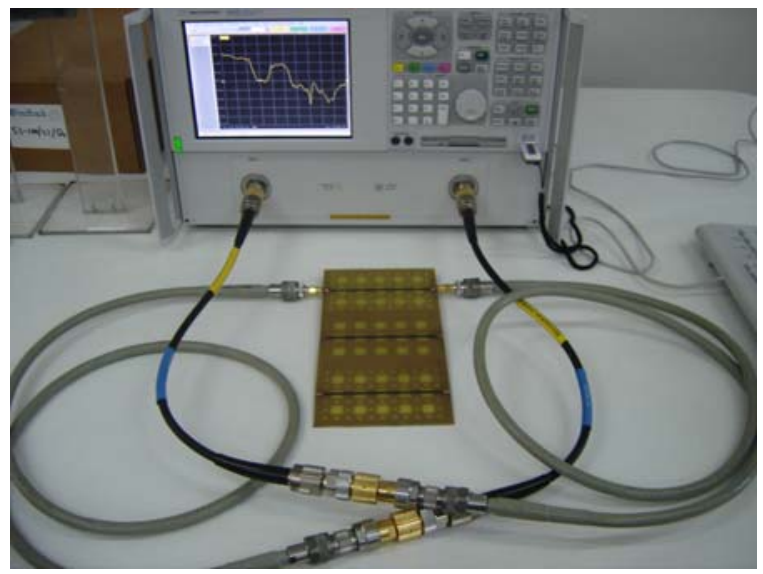

Fig. 8. Set up de medição dos filtros de linha de microfita com plano terra fractal.

\section{RESULTADOS MEDIDOS E SIMULADOS}

A seguir são discutidos os resultados obtidos para o filtro de linha de microfita com plano de terra fractal sob substrato de fibra de vidro (protótipo 1). Para fins de comparação, os resultados das três medições são apresentados na Fig. 9, para cada iteração fractal. As curvas resultantes são comparadas com uma curva de referência, que diz respeito à medição da estrutura com plano de terra completo, ou seja, sem a presença de geometria fractal (nível zero), o que indica as perdas de inserção inerentes à medição. Na Fig. 10, são mostradas as respostas dos filtros com diferentes iterações fractais, excluídas as perdas de inserção medidas para o nível zero de referência. A resposta do filtro de nível 1 indica que este funcionou estritamente como um filtro passa-baixa, com uma freqüência de corte (ponto de $-3 \mathrm{~dB}$ ) em 2,9 GHz, exibindo um comportamento típico dos filtros de Chebyshev, com ripple na banda passante e alta seletividade em freqüência. Os filtros construídos com fractais de níveis 2 e 3 apresentaram comportamentos de filtros passa-faixas com as seguintes bandas passantes: 0,0 - 2,6 GHz e 5,7 - 6,3 GHz (2 $2^{\mathrm{a}}$ iteração), e 0,0 - 3,0 GHz e 5,8 - 6,1 GHz (3 ${ }^{\text {a }}$ iteração). Por outro lado, a partir da Fig. 10, observa-se que o filtro de nível 1 apresenta maior perda de inserção para freqüências abaixo de $6,0 \mathrm{GHz}$, enquanto que os filtros de níveis 2 e 3 apresentam maior perda de inserção acima de 7,5 GHz, com valores abaixo de $-15 \mathrm{~dB}$.

A Fig. 11 mostra resultados das medições realizadas com o segundo protótipo construído (Fig. 7). Neste caso, foi medida a perda de retorno (com a linha de microfita terminada em circuito aberto, funcionando como uma antena) para três comprimentos distintos da fita condutora de excitação, a qual está colocada no lado oposto ao do arranjo fractal. A largura da fita condutora é $w=4 \mathrm{~mm}$ e os três comprimentos adotados para a fita foram $\mathrm{L}_{1}=6 \mathrm{~mm}, \mathrm{~L}_{2}=12 \mathrm{~mm} \mathrm{e} \mathrm{L}_{3}=18$ mm. Observou-se que a diminuição do comprimento da fita resulta em um aumento na freqüência de ressonância da estrutura, assim como da largura de banda (aproximadamente $100 \mathrm{MHz}$ para $\mathrm{L}_{2}$ e $200 \mathrm{MHz}$ para $\mathrm{L}_{3}$ ).

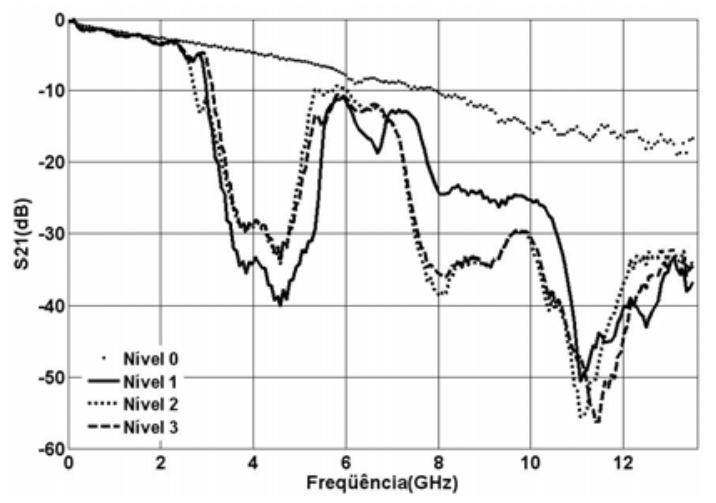

Fig. 9. Comparação dos resultados medidos com o nível zero de referência.

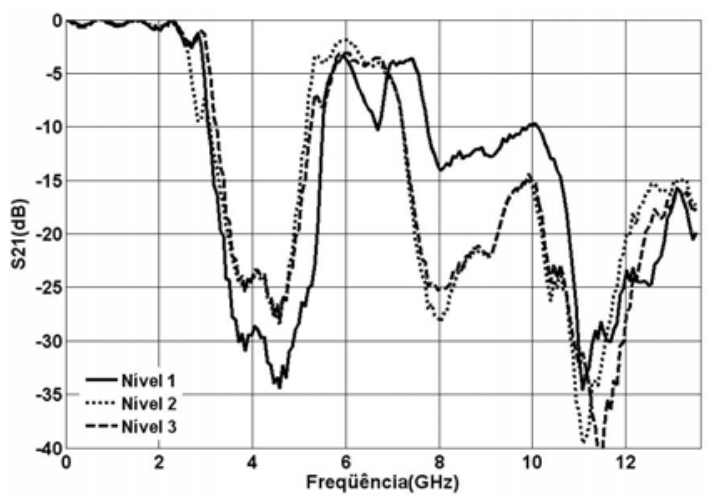

Fig. 10. Subtração dos resultados medidos com o nível zero de referência.

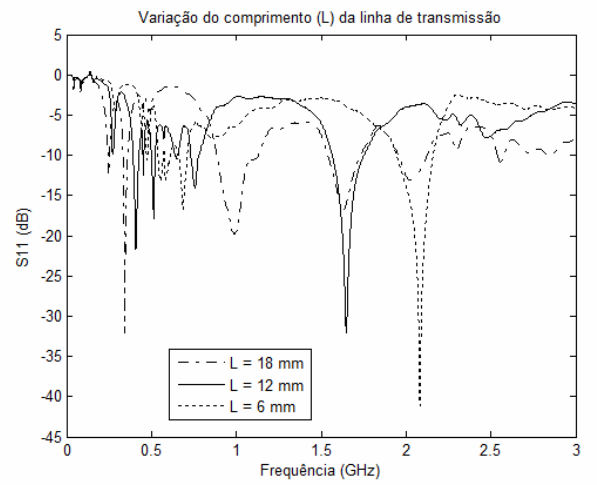

Fig. 11. Perda de retorno para diferentes comprimentos da fita condutora. 


\section{CONCLUSÕES}

Neste trabalho, foi efetuada uma investigação das propriedades de filtros de linhas de microfita com plano de terra fractal, sendo construídos vários protótipos para fins de medição. Das respostas em freqüência medidas para o parâmetro de transmissão $\left(\mathrm{S}_{21}\right)$ e para o parâmetro de reflexão $\left(S_{11}\right)$, verificou-se que os filtros apresentaram um comportamento multibanda.

Os filtros referentes à Fig. 4 (iterações 1, 2 e 3) possuem respostas similares a de filtros de Chebyshev, apresentando um pequeno valor de ripple na banda passante abaixo de $3 \mathrm{GHz}$ e alta seletividade em freqüência. O filtro de nível 1 apresentou uma resposta típica de filtro passa-baixa. Já as respostas correspondentes aos filtros de nível 2 e 3 correspondem a respostas de filtros passa-faixas. Portanto, estes filtros podem ser usados para o acoplamento de sinais em 2,4 GHz e 5,8 GHz, que são duas freqüências bastante utilizadas na banda ISM, pelos padrões IEEE 802 a, b e g, além de aplicações para WIMAX, por exemplo.

Com relação à segunda estrutura considerada, as respostas em freqüência obtidas para a perda de retorno permitem concluir que ela apresenta um comportamento similar ao de uma antena multibanda, sendo observado um aumento nas freqüências de ressonância, à medida que é diminuído o comprimento da fita condutora de excitação. A resposta em freqüência da perda de retorno também permite concluir que existe uma relação de proporcionalidade entre o aumento da complexidade da estrutura e o aumento da largura de banda apresentada. Na continuidade do trabalho, pretende-se investigar a utilização da geometria fractal como plano de terra em filtros de linhas acopladas, como forma de otimização destes circuitos em sistemas de telecomunicações. Uma outra proposta seria investigar os efeitos da alteração da escala do fractal, através do uso de procedimentos para dimensionar o fractal, de acordo com os resultados desejados.

\section{REFERÊNCIAS}

[1] A. A. Kishk, K. F. Lee, W. C. Mok e K. M. Luk, "A wide-band small size microstrip antenna proximately coupled to a hook shape probe". IEEE Trans. Ant. Propag., vol. 52, n. 1, pp. 59-65.

[2] S. Reed, L. Desclos, C. Terret e S. Toutain, "Patch Antenna Size Reduction by Means of Inductive Slots", Microwave and Optical Technology Letters, v. 29, N2, Abril 2001.

[3] J. P. Gianvittorio e R. S. Yahya, "Fractal antennas: a novel antenna miniaturization technique, and applications”, IEEE Trans. Antennas and Propagation Magazine, v. 5, N1, Fevereiro 2002.

[4] L. F. Costa e A. G. C. Bianchi, "A outra dimensão da dimensão fractal”, Revista Ciência Hoje, v. 31, n. 183, p. 40-47, jun. 2002.

[5] K. Falconer, "Fractal geometry: mathematical foundations and application”, 2. ed. Londres: Wiley \& Sons, Ltd., 2003. 337p.

[6] B. B. Mandelbrot, "The fractal geometry of nature”, 3. ed. Nova York: W. H. Freeman and Co., 1982. 468p.

[7] E. H. Fooks e R. A. Zakarevicius, Microwave Engineering Using Microstrip Circuits. Prentice Hall, 1990.

[8] M. G. Passos, R. B. Medeiros, R. B. Carvalho e P. H. F. da Silva, "Projeto, fabricação e medição de filtros rejeita-faixa utilizando estruturas periódicas PBG-GP e superfícies seletivas em freqüência”. In: Congresso de Pesquisa e Inovação da Rede Norte-Nordeste de Educação Tecnológica, 1, 2006, Natal: CEFET-RN. 1 CD-ROM. 